\title{
Transient chaotic transport in dissipative drift motion
}

\author{
R. S. Oyarzabal ${ }^{1,2}$, J. D. Szezech Jr${ }^{3}$, A. M. Batista ${ }^{3,4}$, S. L. T. \\ ${\text { de } \text { Souza }^{5} \text {, I. L. Caldas }}^{4}$, R. L. Viana ${ }^{6}$, M. A. F. Sanjuán ${ }^{2}$ \\ 1 Pós-Graduação em Ciências/Física, Universidade Estadual de Ponta Grossa, \\ 84030-900, Ponta Grossa, PR, Brazil \\ 2 Departamento de Física, Universidad Rey Juan Carlos, Tulipán s/n, 28933 \\ Móstoles, Madrid, Spain \\ 3 Departamento de Matemática e Estatística, Universidade Estadual de Ponta \\ Grossa, 84030-900, Ponta Grossa, PR, Brazil \\ ${ }^{4}$ Instituto de Física, Universidade de São Paulo, 05315-970, São Paulo, SP, Brazil \\ ${ }^{5}$ Departamento de Física e Matemática, Universidade Federal de São João del Rei, \\ 36420-000, Ouro Branco, MG, Brazil \\ 6 Departamento de Física, Universidade Federal do Paraná, 81531-990, Curitiba, PR, \\ Brazil
}

\begin{abstract}
We investigate chaotic particle transport in magnetized plasmas with two electrostatic drift waves. Considering dissipation in the drift motion, we verify that the removed KAM surfaces originate periodic attractors with their corresponding basins of attraction. We show that the properties of the basins depend on the dissipation and, consequently, the space-averaged escape time decays exponentially when the dissipation increases. We find positive finite time Lyapunov exponents in dissipative drift motion, consequently the trajectories exhibit transient chaotic transport. These features support the understanding of plasma confinement properties considered with a dissipative system.
\end{abstract}

Keywords: escape time, transport, drift waves

PACS numbers: 52.35.Kt,05.45.Ac

Submitted to: J. Phys. A: Math. Gen. 


\section{Introduction}

It is well known that diamagnetic drifts are expected when a magnetically confined plasma displays density gradients [1]. Drift waves are electrostatic in low-beta plasmas, with fluctuations in density, floating potential and temperature [2]. They have relatively long wavelength (compared with the ion Larmor radius), what makes them good candidates for explaining anomalous diffusion across magnetic fields [3]. Nearly coherent drift waves are usually seen in linear machines and drift wave turbulence in toroidal plasmas [4].

Drift waves play a relevant role in transport of particles in magnetically confined plasmas. In fact, the presence of steep density gradients in plasma edge gives rise to turbulence, that can clear up anomalous transport rates observed experimentally. A theoretical description of drift waves driven transport was proposed by Horton leading to a model using partial differential equations for the electrostatic potential $[5,6]$. Consequently, drift waves appear in magnetized plasmas producing the dominant mechanism for transport of particles across magnetic field lines. A large amount of information obtained from quasi-stationary laboratory experiments for plasma confinement has been reviewed for drift waves, which become unstable by density gradients, temperature gradients and trapped particle effects $[3,7]$.

Qualitative characteristics of the transport can be approximated by a lowdimensional dynamical system with island chains in phase space due to the superposition of two dominant drift waves [8]. We consider here a two wave Hamiltonian model which is the simplest system of waves that can exhibit chaotic behaviour used to describe drift waves in magnetic confinement devices and space plasmas [5, 9]. Therefore, the chaotic transport of plasma particles are typically related to anomalous diffusion [10]. In addition to the two-wave Hamiltonian model, we have included a dissipation term to study the transport and escape time in dissipative drift motion.

The effect of dissipation on Hamiltonian systems has been an important topic of research. For instance, studies about chaotic scattering in nonlinear physics such as nuclear physics, optical systems, and particle advection in hydrodynamical flows have been described in [11]. Furthermore, the effect of weak dissipation can have relevant consequences on the chaotic scattering as shown in $[12,13]$. In addition, fractal structures in phase space $[14,15]$ have been investigated in a dissipative chaotic scattering model in [16]. They have verified that the variation of the fractal dimension as a function of the dissipation parameter is described by a crossover phenomenon. There have been further studies about the phase space basin structure of chaotic scattering problems in Hamiltonian systems in [17, 18, 19, 20]. Another interesting aspect concerns the observation that the Wada basin topology remains qualitatively unchanged for nonhyperbolic chaotic scattering when a weak dissipation is included in a Hamiltonian system [12]. Dissipation also limits the particle acceleration, excluding the Fermi acceleration found in some Hamiltonian systems [21]. It is interesting to note that the dissipation may be associated with collisional effects of particles in confined 
plasmas [22] or even the energy loss through emission of radiation (since gyrating charged particles do so).

The presence of multiple coexisting attractors, or multistability, is also expected in Hamiltonian systems with weak dissipation. In this case, numerical and rigorous analyses point out that there is a large number of coexisting periodic attractors [23]. For instance, center fixed points of the conservative system (neutrally stable) become, with a small amount of dissipation, stable foci. If such points belong to a chain of $m$ periodic islands, they become period- $m$ foci [24]. As a general rule, the number of attractors tends to infinity as the dissipation vanishes [25]. For small yet nonzero damping, we expect only a finite number of coexisting attractors. This occurs because many periodic orbits (particularly those with high periods) lose their stability in a very rapid way as damping grows up [26].

In this work, we investigate the dynamical consequences of introducing dissipation in plasmas by using a two-wave model and where dissipation can cause the destruction of the KAM tori and the chaotic region. As a result, attractors begin to appear in phase space and consequently their corresponding basins of attraction. Moreover, we observe that the pattern of the basin of attraction has a dependence on the damping. We also analyze the particle escape time, and our results show that the space-averaged escape time obeys a power-law scaling with the damping constant. Finally, we have verified the existence of transient chaotic transport.

This paper is organized as follows: in Section 2 we introduce the drift waves model. Section 3 exhibits our results about basins of attractions. In the same phase space we observe that the periodic orbits become periodic attractors. In Section 4, we show the escape time and the transient chaotic transport. The time that a particle stay in a region depends on the magnitude of dissipation. We show also that the space-averaged escape time as a function of the damping is represented as the a power-law decay. To verify the transient chaotic transport we compute the finite time Lyapunov exponents. Finally, in the last section, we draw the conclusions.

\section{Drift waves model}

We introduce here the two-wave Hamiltonian particle transport induced by electrostatic waves propagating in the poloidal direction in a magnetized plasma with a constant toroidal magnetic field $\vec{B}=B_{0} \hat{e}_{z}$. The drift velocity of the guiding centers is given by $\vec{v}=(\vec{E} \times \vec{B}) / B^{2}$, and we assume particles moving at this velocity. The electric field is given by the gradient of the potential in the plasma edge $\vec{E}=-\nabla \phi(x, y, t)$, where $\phi$ is an electrostatic potential given by

$$
\phi(x, y, t)=\phi_{0}(x)+\sum_{i=1}^{N} A_{i} \sin \left(k_{x_{i}} x\right) \cos \left(k_{y_{i}} y-\omega_{i} t\right) .
$$

This function is composed of a background equilibrium electrostatic potential, given by $\phi_{0}(x)=a x$, with the superposition of a collection of $N$ drift waves propagating in the 
poloidal direction $y$. Consequently, the equations of motion are

$$
\begin{aligned}
& \dot{x}=-\frac{1}{B_{0}} \frac{\partial \phi}{\partial y}, \\
& \dot{y}=\frac{1}{B_{0}} \frac{\partial \phi}{\partial x} .
\end{aligned}
$$

Defining $H(x, y, t)=\phi / B_{0}$, and identifying $x$ as the canonical momentum and $y$ as its conjugate coordinate, we can see that equation (2) is a Hamiltonian system. For large aspect ratio tokamaks, we use the Cartesian coordinates and identify $x$ and $y$ as the radial and poloidal coordinates, respectively.

Introducing the variables

$$
\begin{aligned}
& x^{\prime}=\frac{x}{r_{0}}, y^{\prime}=\frac{y}{r_{0}}, a^{\prime}=\frac{a}{E_{0}}, t^{\prime}=\frac{t}{t_{0}}, \\
& \omega^{\prime}=\omega_{i} t_{0}, u^{\prime}=\frac{u}{v_{0}}, A_{i}^{\prime}=\frac{A_{i}}{E_{0} r_{0}}, k_{i}^{\prime}=\frac{\omega^{\prime}}{u^{\prime}}=k_{i} r_{0},
\end{aligned}
$$

we can find the dimensionless Hamiltonian [5]

$$
H(x, y, t)=a x+\sum_{i=1}^{N} A_{i} \sin \left(k_{x_{i}} x\right) \cos \left(k_{y_{i}} y-\omega_{i} t\right) .
$$

For $N=1$ the system is integrable and presents stable orbits, whereas for $N>1$ the integrability depends on the phase of each wave velocity. The system is integrable when the phase velocities are the same. However, the system is not integrable and can exhibit chaotic behaviour, if at least one phase velocity is different.

Here, we consider the trajectories driven by a resonant wave whose phase velocity $u_{1}=\omega_{1} / k_{y_{1}}=a$, where $a$ is a constant. Trajectories are trapped in phase space in cells separated by a separatrix. The addition of a perturbing second wave breaks the separatrix and the remaining cells become immersed in a chaotic sea. With these considerations in mind, the equations of motion can be derived from the Hamiltonian

$$
H(x, y, t)=A_{1} \sin \left(k_{x 1} x\right) \cos \left(k_{y 1} y\right)+A_{2} \sin \left(k_{x 2} x\right) \cos \left(k_{y 2}(y-u t)\right),
$$

where $u=\left|\omega_{2} / k_{y 2}-\omega_{1} / k_{y 1}\right|$ is the difference of phase velocity between the two waves. We add damping terms to the equations of motion derived from (5) obtaining

$$
\begin{aligned}
\frac{d x}{d t}= & A_{1} k_{y 1} \sin \left(k_{x 1} x\right) \sin \left(k_{y 1} y\right)+ \\
& A_{2} k_{y 2} \sin \left(k_{x 2} x\right) \sin \left[k_{y 2}(y-u t)\right]-\mu_{x} x, \\
\frac{d y}{d t}= & A_{1} k_{x 1} \cos \left(k_{x 1} x\right) \cos \left(k_{y 1} y\right)+ \\
& A_{2} k_{x 2} \cos \left(k_{x 2} x\right) \cos \left[k_{y 2}(y-u t)\right]-\mu_{y} y,
\end{aligned}
$$

where the parameters $\mu_{x}$ and $\mu_{y}$ are the damping constants, and we consider $\mu_{x}=\mu_{y}=\mu$ in this work.

Without damping, single particle motion in one drift wave is described by an integrable Hamiltonian system and consequently it can be solved analytically. For a resonant wave, a two-dimensional lattice of counter rotating rolls separated by a 


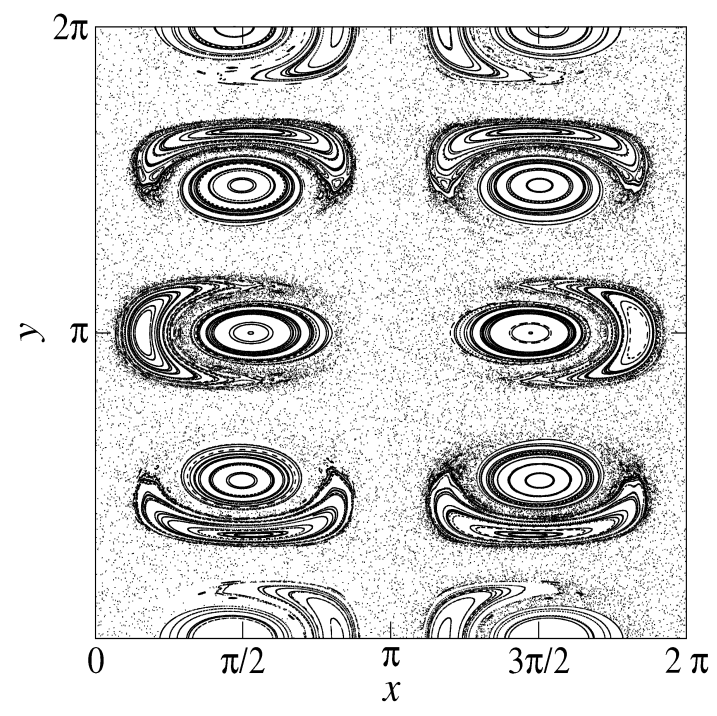

Figure 1. Poincaré section of the phase space plot for $A_{1}=1, A_{2}=0.1, k_{x 1}=1$, $k_{x 2}=\sqrt{3}, k_{y 1}=1, k_{y 2}=0.5, u=1.5$, and $\mu=0$.

separatrix is created in the resonant region. The particles cannot cross the separatrix so that they are confined to motion within a single roll. The second wave, with an amplitude smaller than that of the first wave, is treated as a perturbation. The Hamiltonian is no longer time-independent so that a particle is no longer confined to a single roll [3]. Then, qualitative features of the transport can be approximated by a lowdimensional dynamical system with island chains in phase space due to the superposition of two dominant drift waves.

Figure 1 shows a Poincaré cross-section, where we consider $A_{1}=1, A_{2}=0.1$, $k_{x 1}=1, k_{x 2}=\sqrt{3}, k_{y 1}=1, k_{y 2}=0.5, u=1.5$, and $\mu=0$. The Poincaré cross-section is obtained by means of the values of the motion sampled at each time period given by $2 \pi / u$. We can see periodic orbits as well chaotic behaviour which can be found around them. The phase space of a Hamiltonian system generally consists of islands of invariant tori immersed in a chaotic sea connecting the main islands. Consequently, in the considered phase space the chaotic particles are not confined.

\section{Basins of attraction}

A basin of attraction is a region of phase space defined by the set of points that taken as initial conditions are driven by the dynamics of a dissipative dynamical system to a particular attractor. In other words, in dynamical systems, an attractor is a set towards that a system tends to evolve, regardless of the starting conditions inside its basin of attraction [27]. Property values that get close enough to the attractor values remain close even if slightly disturbed.

Figure 2 exhibits, in black points, the appearance of periodic orbits for the dissipation free case $\mu=0$ (conservative). This structure does not persist if a small 


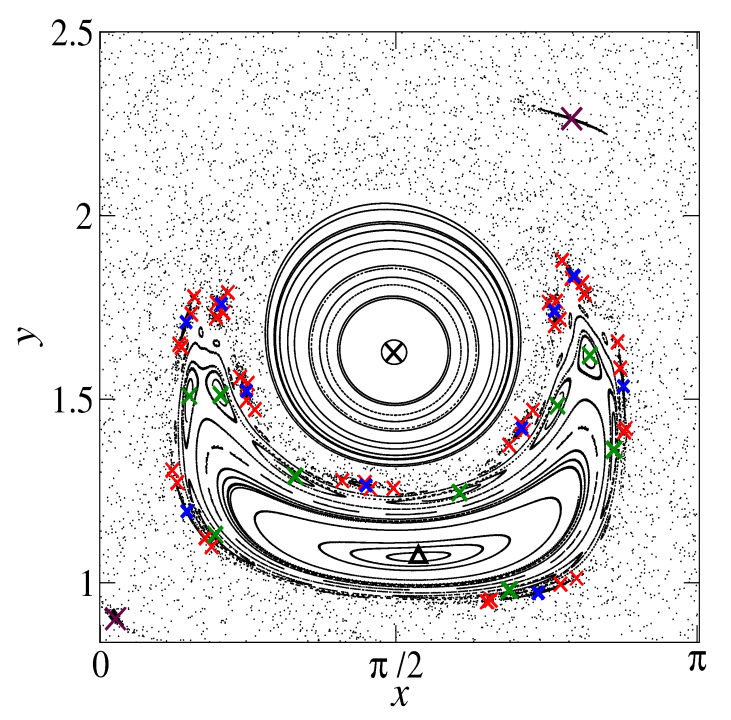

Figure 2. (Colour online) Poincaré section of the phase space plot for $A_{1}=1$, $A_{2}=0.1, k_{x 1}=1, k_{x 2}=\sqrt{3}, k_{y 1}=1, k_{y 2}=0.5$, and $u=1.5$. The black points correspond to the dissipation free case, $\mu=0$, and the coloured symbols to the case with a small amount of dissipation $\mu=10^{-3}$.

amount of dissipation is introduced, since the periodic orbits become attractors. We can see some attractors that are identified by coloured symbols, where we consider a small amount of dissipation, $\mu=10^{-3}$. There are two period-1 attractors, one represented by a black cross and another by a black triangle. The brown crosses correspond to a period-2 attractor. The green crosses represent a period-3 attractor. The attractors with period 10 and with period 40 are identified by blue and red crosses, respectively.

We can see basins of attraction in figure 3 for the cases (a) $\mu=10^{-3}$, (b) $\mu=10^{-2}$, and (c) $\mu=10^{-1}$. The procedure used to obtain the basin of attraction consists of iterating a grid of initial conditions in phase space and observing their asymptotic behaviour. The black regions represent the initial conditions that go to one period-1 attractor, and the red regions correspond to the other period-1 attractor. The yellow regions represent the initial conditions that escape outside this phase space region. It is possible to see small white regions that are attractors of period greater than one. For a weak damping the system presents a high number of coexisting attractors, and its corresponding basin has a complex structure. By increasing the value of the damping it is possible to verify a decrease in the number of attractors. Due to the dissipation, the chaotic motion is confined in phase space. Even so, the chaotic trajectories escape from certain regions, as discussed in the next section.

Chaotic behaviours begins with the presence of the second wave for the dissipative free case. The phase space in figure 2 is composed of chaotic and stable orbits, and furthermore the system displays anomalous transport [5]. A closer real system imply loss of particles and energy in the plasma edge. The $\vec{E} \times \vec{B}$ drift motion is a simple model to study fusion and confinement in plasma as well as transport properties. Attractors 

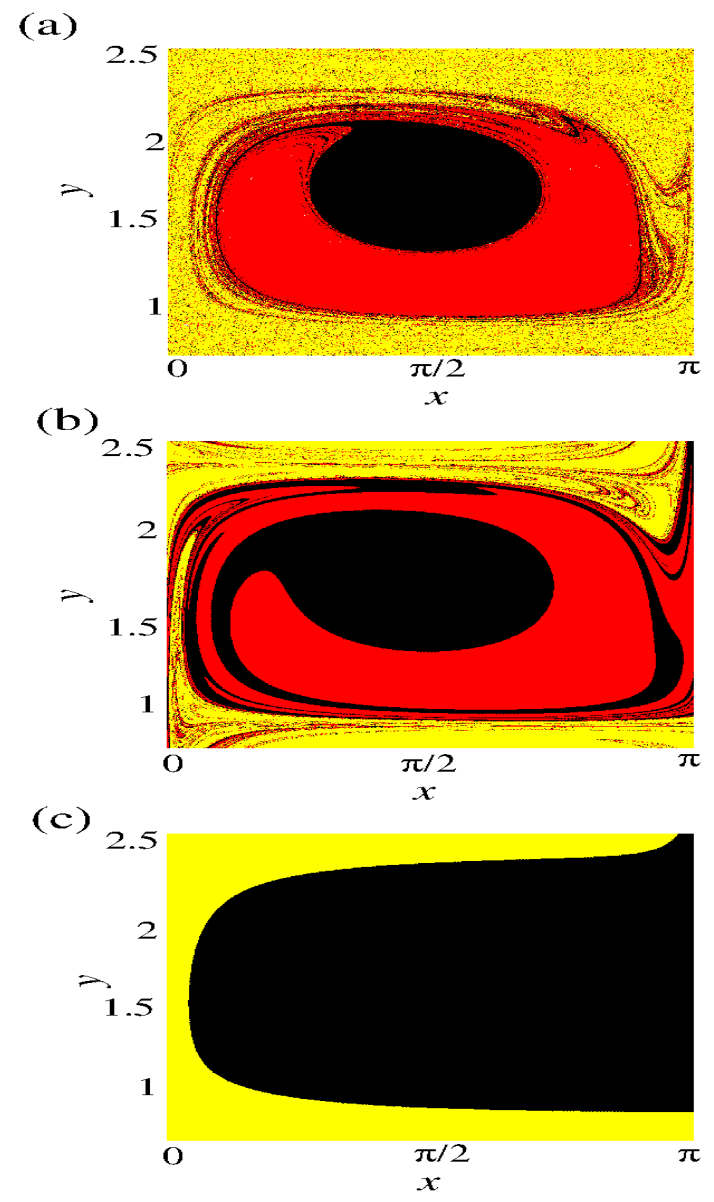

Figure 3. (Colour online) Basin attraction for (a) $\mu=10^{-3}$, (b) $\mu=10^{-2}$, and (c) $\mu=10^{-1}$. The black and red colours correspond to the basins of period-1 attractors and the yellow colour corresponds to the initial conditions that escape outside this phase space region.

and their corresponding basins of attraction have never been considered in the two waves model. In the dissipative case of figure 2 there are several attractors represented with coloured symbols, however not all initial conditions in the grid go to these attractors. This is shown in figure 3(a) with dissipation $\mu=10^{-3}$, where the yellow color represents initial conditions that escape out of the bounded region. We set a limit on the system and we proved that some particles tend to go to periodic attractors. We may think that others particles can be attracted to others attractors off the grid or hit to the tokamak walls.

Chaotic orbits in figure 2 are in the same region where in figure 3(a) black, red and yellow points are mixed. In the place where there were chaotic orbits in the dissipative free case there is no well defined basins of attraction, although in these regions it seems that the yellow initial conditions are more predominant. On the other hand, it is possible to determine a basin of attraction composed of black and red points. Such particles which go to period-1 attractors were periodic orbits in the conservative system. This 
(a)

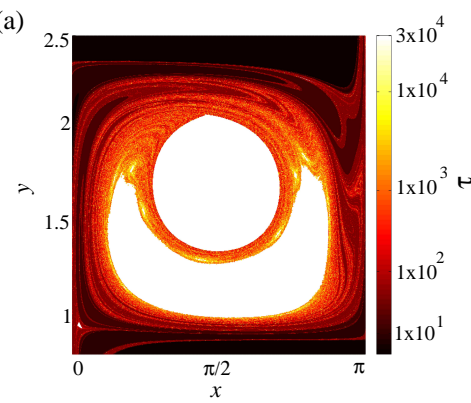

(c)

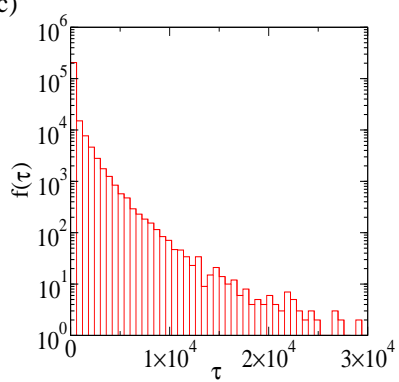

(b)

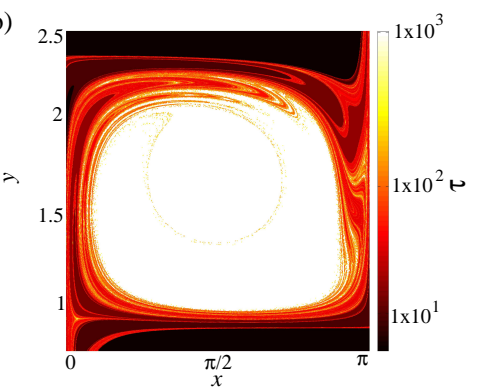

(d)

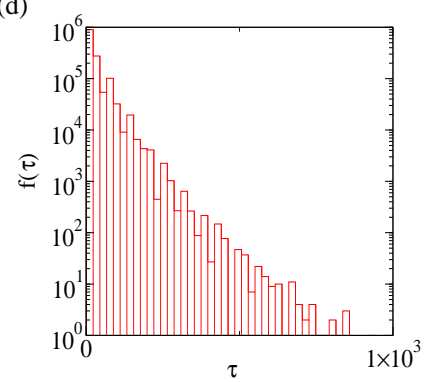

Figure 4. (Colour online) Escape time in colour scale as a function of the initial conditions for (a) $\mu=10^{-5}$ and (b) $\mu=10^{-3}$. (c) and (d) are the distributions of the escape time corresponding to (a) and (b), respectively.

way, with the small dissipation is not possible to predict which initial conditions in the chaotic sea will go to an attractor or which escape for the plasma edge. In figure 3(b) damping is increased and the limit of the black and red basins start to blend. At the same time the yellow region start to stay more defined. We can see regions where there are not mixed points, in addition yellow homogeneous regions are formed.

The scenery changes with dissipation $\mu=10^{-1}$. The period-1 attractor of the red basin disappears. We have now a basin of attraction composed of black and yellow points. In figure 3(c) the basin of attraction represented by the yellow color does not share the same regions of the others points which go to an attractor, instead there is a basin of attraction defined for the particles that go out the bounded region. This way, we have a basin of attraction of particles which can escape, can be for attractors off the grid or will bumps into the tokamak walls. Even with dissipation, it is possible to observe a trapped effect, because particles are attracted for regions in the plasma, and other particles escape towards the plasma edge. Thereby, we observe that transport properties change in addition of dissipation in drift $\vec{E} \times \vec{B}$ motion.

\section{Escape time and transient chaotic transport}

Our goal here is to study the dependence of the escape time on the damping parameter. We consider that a trajectory has escaped when it has crossed the boundary of the phase space region. In order to obtain the passage time for the escape we consider the phase space region $0<x \leq \pi$ and $0.7<y \leq 2.5$. 


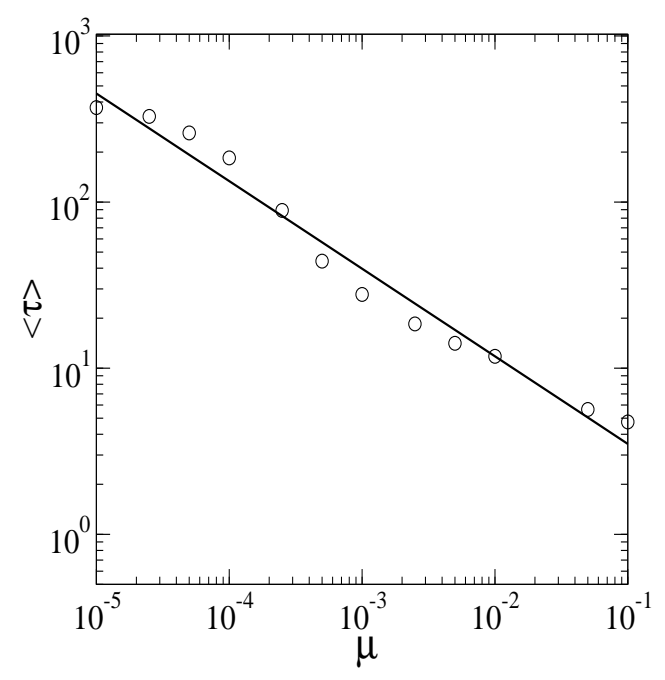

Figure 5. Space-averaged escape time as a function of the damping constant. The solid line is a power-law fit.

Figure 4 presents the escape time for $A_{1}=1, A_{2}=0.1, k_{x 1}=1, k_{x 2}=\sqrt{3}, k_{x 1}=1$, $k_{y 2}=0.5$, and $u=1.5$. In figure4(a) and (b) we show the escape time $(\tau)$ in colour scale from a grid of initial conditions under the drift waves model with dissipation equal to $\mu=10^{-5}$ and $\mu=10^{-3}$, respectively. The white region are the initial conditions which do not escape from the phase space region, namely there are initial conditions which go to an attractor there. We compute the distribution $f(\tau)$, shown in figure 4(c) $\left(\mu=10^{-5}\right)$ and $(\mathrm{d})\left(\mu=10^{-3}\right)$, by means of the escape times $\tau$ of initial conditions outside the basin of attraction. In this case, the distributions are skewed right, due to the fact that a high quantity of initial conditions have an escape time larger than $10^{4}$ for $\mu=10^{-5}$, and larger than $10^{2}$ for $\mu=10^{-3}$.

In order to verify the behaviour of the escape time with the damping, we calculate the space-averaged escape time

$$
<\tau>=\frac{1}{M} \sum_{m=1}^{M} \tau_{m},
$$

where $M$ is the number of initial conditions that escape from the phase space region. The dependence of the space-averaged escape time upon the damping is depicted in figure 5 , where the mean value is calculated by means of $2.5 \times 10^{5}$ initial conditions. The leastsquares fit in figure 5 shows a power-law dependence on the form $\langle\tau\rangle=1.04 \mu^{-0.53}$. This way, the space-averaged decreases when the damping increases. The decay of the transient trajectories can be associated to the homoclinic tangle present in the chaotic sea, as reported for chaotic field lines escape in tokamaks [28].

Transport phenomena is relevant for many applications such as fluid mixing, particle accelerators, and particle transport in fusion plasmas [29]. The focus of such studies on 
magnetic fusion have been on the transport processes in magnetically confined plasma [30, 31]. Marcus and collaborators [32] studied chaotic transport in reversed shear plasma profiles, where they investigated effects from electric and magnetic reversed shear fields on plasma confinement. Chaotic transport occurs when the particle trajectories present chaotic behaviour. In figure 1, due to the chaotic sea, it is possible to observe the chaotic transport. Nevertheless, the chaotic sea disappears when the damping is introduced.

Lyapunov exponents are a good diagnostic tool for analyzing chaotic motion. Studies about finite-time Lyapunov exponents in nonlinear physics as shown in [33, 34]. Finite Lyapunov exponents allow the understanding of local and global properties of a dynamical flow, as well as nonhyperbolic behavior. This way, we analyze the particle trajectories by means of the Lyapunov exponents in dissipative drift motion.

The $i$ th Lyapunov exponent is defined in terms of the growth rate of the $i$ th principal axis of the ellipsoid $p_{i}(t)$ monitored of an infinitesimal $n$-sphere of initial conditions,

$$
\lambda_{i}=\lim _{t \rightarrow \infty} \frac{1}{t} \log \frac{p_{i}(t)}{p_{i}(0)} .
$$

The system is chaotic when it has one or more positive Lyapunov exponents. In our simulations, the Lyapunov exponents are obtained according to the algorithm proposed by Wolf et al. [35]. Next, to analyze transient chaos we calculate Lyapunov exponent for finite time intervals [36]. The $i$ th $(i=1,2)$ time- $T$ Lyapunov exponent associated with the point $(x(0), y(0))$ is given by

$$
\lambda_{i}(x(0), y(0), T)=\frac{1}{T} \ln \left(\left\|D \mathcal{M}^{T}(x(0), y(0)) \mathcal{U}_{T}\right\|\right),
$$

where $D \mathcal{M}^{T}(x(0), y(0))$ denotes the Jacobian matrix of the time interval $T$ evaluated at the point $(x(0), y(0))$, and $\mathcal{U}_{T}$ is the eigenvector. The finite time Lyapunov exponents depend on the initial condition $(x(0), y(0))$, while their infinite time counterparts

$$
\lambda_{i}=\lim _{T \rightarrow \infty} \lambda_{i}(x(0), y(0), T)
$$

present the same value for almost every point $(x(0), y(0))[37]$.

Attractors appear when we introduce damping in the drift motion, consequently there are no islands neither chaotic sea in the phase space. Having this in mind, we used the finite time Lyapunov exponents [36] to verify the existence of transient chaotic transport. We consider that this form of transport occurs before the particle trajectories go to the periodic attractors. Figure 6 shows the finite time Lyapunov exponents in colour scale as a function of the initial conditions. The dark blue regions correspond to the particle trajectories that do not escape to attractors outside the region $0 \leq x \leq \pi$ and $0.5 \leq y \leq 2.5$. The regions with colours different of dark blue represent the trajectories that not only escape from this region, but also have positive finite time Lyapunov exponents. As a result, in these regions the trajectories present transient chaotic transports. 


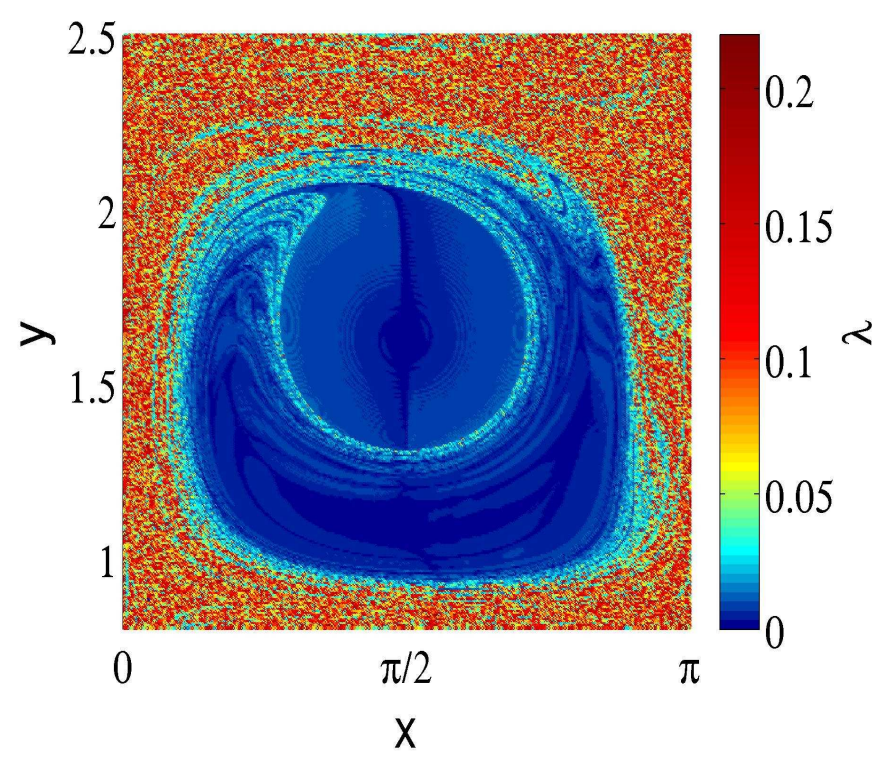

Figure 6. (Colour online) Finite time Lyapunov exponents in colour scale as a function of the initial conditions for $T=100, A_{1}=1, A_{2}=0.1, k_{x 1}=1, k_{x 2}=\sqrt{3}, k_{y 1}=1$, $k_{y 2}=0.5, u=1.5$, and $\mu=0.001$.

\section{Conclusions}

We have analyzed a two-wave Hamiltonian model. The model describes a chaotic transport of particles in plasma confinement. The chaotic transport of plasma particles are typically related to anomalous diffusion.

We have included a dissipative term in the two-wave model. With the dissipation we were able to observe the destruction of KAM tori, as well as the disappearance of the chaotic region. Due to the damping, the system possess basin of attraction. Furthermore, we verified that the pattern of the basin of attraction depends on the damping constant.

For the damped two-wave model, we have investigated the escape time dependence on the damping constant. We have observed that the quantity of initial conditions with high escape time decreases when the damping constant increases. Moreover, our numerical results show that the space-averaged escape time obeys a power-law scaling with the increasing of the damping. We have also studied the transport and we have identified transient chaotic transport.

\section{Acknowledgments}

This work was made possible through partial financial support from the following Brazilian research agencies: CNPq, CAPES, FAPESP, and Fundação Araucária. Financial support by the Spanish Ministry of Economy and Competitiveness under project number FIS2013-40653-P is also acknowledged. 


\section{References}

[1] Chen F F 1984 Plasma Physics and Controlled Fusion (New York: Plenum Press)

[2] Tang W M 1978 Microinstability theory in tokamaks Nucl. Fusion 18 1089-1160

[3] Horton W 1999 Drift waves and transport Rev. Mod. Phys. 71 735-778

[4] Motley R W 1975 Q-Machines (New York: Academic Press)

[5] Horton W 1985 Onset of stochasticity and the diffusion approximation in drift waves Plasma Phys. Contr. F. 27 937-956

[6] Horton W 1990 Nonlinear drift waves and transport in magnetized plasma Phys. Rep. 192 1-177

[7] Horton W 1981 Anomalous ion conduction from toroidal drift modes Plasma Phys. 23 1107-1125

[8] Marcus F A, Caldas I L, Guimarães-Filho Z O, Morrison P J, Horton W, Nascimento I C, Kuznetsov Y K 2008 Reduction of chaotic particle transport driven by drift waves in sheared flows Phys. Plasmas 15112304

[9] Kleva R G, Drake J F 1984 Stochastic ExB particle transport Phys. Fluids 271686

[10] Alligood K T, Sauer T D, Yorke J A 1997 Chaos: An introduction to dynamicl systems (New York: Springer-Verlag)

[11] Seoane J M, Sanjuán M A F 2013 New developments in classical chaotic scattering Rep. Prog. Phys. 760116001

[12] Seoane J M, Aguirre J, Sanjuán M A F 2006 Basin topology in dissipative chaotic scattering Chaos 16023101

[13] Bleza F, Seoane J M, Barrio R, Sanjuán M A F 2012 To escape or not to escape, that is the question: Perturbing the Hénon-Heiles Hamiltonian Int. J. Bifurcation Chaos 221230010

[14] Viana R L, Silva E C, Kroetz T, Caldas I L, Roberto M, Sanjuán M A F 2011 Fractal structures in nonlinear plasma physics Phil. Trans. R. Soc. A 369 371-395

[15] Aguirre J, Viana R L, Sanjuán M A F 2009 Fractal structures in nonlinear dynamics Rev. Mod. Phys. 81 333-386

[16] Seoane J M, Sanjuán M A F, Lai Y C 2007 Fractal dimension in dissipative chaotic scattering Phys. Rev. E $\mathbf{7 6} 016208$

[17] Bernal J D, Seoane J M, Sanjuán M A F 2013 Weakly noisy chaotic scattering Phys. Rev. E 88 032914

[18] Seoane J M, Huang L, Sanjuán M A F, Lai Y C 2009 Effect of noise on chaotic scattering Phys. Rev. E 79047202

[19] Bleza F, Seoane J M, Barrio R, Sanjuán M A F 2014 Effects of periodic forcins in chaotic scattering Phys. Rev. E $\mathbf{8 9} 042909$

[20] Oliveira D F M, Leonel E D 2013 Some dynamical properties of a classical dissipative bouncing ball model with two nonlinearities Phys. A 392 1762-1769

[21] Ladeira D G, Leonel E D 2015 Dynamics of a charged particle in a dissipative Fermi-Ulam model Commun. Nonlinear Sci. Numer. Simulat. 20 546-558

[22] Schelin A B, Caldas I L, Viana R L, Benkadda S 2011 Collisional effects in the tokamap Phys. Lett. A 376 24-30

[23] Feudel U, Grebogi C 1997 Multistability and the control of complexity Chaos 7 597-604

[24] Feudel U, Grebogi C, Poon L, Yorke J A 1998 Dynamical properties of a simple mechanical system with a large number of coexisting periodic attractors Chaos Soliton Fract. 9 171-180

[25] Coninck J C P, Lopes S R, Viana R L 2004 Multistability and phase-space structure of dissipative nonlinear parametric four-wave interactions Phys. Rev. E $\mathbf{7 0} 056403$

[26] Feudel U, Grebogi C, Hunt B R, Yorke J A 1996 Map with more than 100 coexisting low-period periodic attractors Phys. Rev. E $\mathbf{5 4} 71-81$

[27] Ott E 2003 Chaos in Dynamical Systems (Cambridge: Cambridge University Press)

[28] Silva E C, Calda I L, Viana R L, Sanjuán M A F 2002 Escape Patterns, Magnetic Footprints, and Homoclinic Tangles Due to Ergodic Magnetic Limiters Phys. Plasmas 9 4917-4928

[29] Benkadda S, Elskens Y, Ragot B 1994 Exit times and chaotic transport in Hamiltonian systems 
Phys. Rev. Lett. 72 2859-2862

[30] Callen J D 1992 Transport processes in magnetically confined plasmas Phys. Fluids B 4 2142-2154

[31] Weiland J 2012 Stability and transport in magnetic confinement systems (New York: Springer)

[32] Marcus F A, Kroetz T, Roberto M, Caldas I L, da Silva E C, Viana R L, Guimarães-Filho Z O 2008 Chaotic transport in reversed shear tokamaks Nucl. Fusion 48024018

[33] Vallejo J C, Viana R L, Sanjuán, M A F 2008 Local predictability and nonhyperbolicity through finite Lyapunov exponent distributions in two-degrees-of-freedom Hamiltonian systems Phys. Rev. E 78066204

[34] Vallejo J C, Aguirre J, Sanjuán, M A F 2003 Characterization of the local instability in the HnonHeiles Hamiltonian Phys. Lett. A 311 26-38

[35] Wolf A, Swift J B, Swinney H L, Vastano J A 1985 Determining Lyapunov exponents from a time series Physica D 16 285-317

[36] Szezech J J D, Caldas I L, Lopes S R, Morrison P J, Viana R L 2012 Effective transport barriers in nontwist systems Phys. Rev. E 86036206

[37] Szezech J J D, Lopes S R, Viana R L 2005 Finite-time Lyapunov spectrum for chaotic orbits of non-integrable Hamiltonian systems Phys. Lett. A 335 394-401 